\title{
BMJ Open Menstrual hygiene management among adolescent girls in India: a systematic review and meta-analysis
}

\author{
Anna Maria van Eijk, ${ }^{1}$ M Sivakami, ${ }^{2}$ Mamita Bora Thakkar, ${ }^{3}$ Ashley Bauman, ${ }^{1}$ \\ Kayla F Laserson, ${ }^{4}$ Susanne Coates, ${ }^{3}$ Penelope A Phillips-Howard ${ }^{1}$
}

To cite: van Eijk AM, Sivakami M, Thakkar MB, et al. Menstrual hygiene management among adolescent girls in India: a systematic review and metaanalysis. BMJ Open 2016;6: e010290. doi:10.1136/ bmjopen-2015-010290

- Prepublication history and additional material is available. To view please visit the journal (http://dx.doi.org/ 10.1136/bmjopen-2015010290).

Received 18 October 2015 Revised 12 January 2016 Accepted 5 February 2016

\section{CrossMark}

\section{${ }^{1}$ Department of Clinical Sciences, Liverpool school of Tropical Medicine, Liverpool, UK \\ ${ }^{2}$ Tata Institute of Social Sciences, School of Health Systems Studies, Mumbai, Maharashtra, India ${ }^{3}$ UNICEF India, New Delhi, India \\ ${ }^{4}$ Centers for Disease Control and Prevention (CDC) India, Atlanta, Georgia, USA}

Correspondence to Anna Maria van Eijk; amvaneijk@gmail.com

\section{ABSTRACT}

Objectives: To assess the status of menstrual hygiene management (MHM) among adolescent girls in India to determine unmet needs.

Design: Systematic review and meta-analysis. We searched PubMed, The Global Health Database, Google Scholar and references for studies published from 2000 to September 2015 on girls' MHM.

\section{Setting: India.}

Participants: Adolescent girls.

Outcome measures: Information on menarche awareness, type of absorbent used, disposal, hygiene, restrictions and school absenteeism was extracted from eligible materials; a quality score was applied. Metaanalysis was used to estimate pooled prevalence (PP), and meta-regression to examine the effect of setting, region and time.

Results: Data from 138 studies involving 193 subpopulations and 97070 girls were extracted. In 88 studies, half of the girls reported being informed prior to menarche (PP $48 \%, 95 \% \mathrm{Cl} 43 \%$ to $53 \%, \mathrm{l}^{2}$ $98.6 \%$ ). Commercial pad use was more common among urban (PP 67\%, 57\% to $76 \%, \mathrm{I}^{2} 99.3 \%, \mathrm{n}=38$ ) than rural girls (PP $32 \%, 25 \%$ to $38 \%, I^{2} 98.6 \%$, $\mathrm{n}=56, \mathrm{p}<0.0001)$, with use increasing over time $(p<0.0001)$. Inappropriate disposal was common (PP 23\%, 16\% to 31\%, $I^{2} 99.0 \%, n=34$ ). Menstruating girls experienced many restrictions, especially for religious activities (PP 0.77, 0.71 to $0.83, \mathrm{I}^{2} 99.1 \%$, $\mathrm{n}=67$ ). A quarter (PP $24 \%, 19 \%$ to $30 \%, \mathrm{I}^{2} 98.5 \%$, $\mathrm{n}=64$ ) reported missing school during periods. A lower prevalence of absenteeism was associated with higher commercial pad use in univariate $(p=0.023)$ but not in multivariate analysis when adjusted for region $(p=0.232, n=53)$. Approximately a third of girls changed their absorbents in school facilities (PP 37\%, $29 \%$ to $\left.46 \%, I^{2} 97.8 \%, n=17\right)$. Half of the girls' homes had a toilet (PP $51 \%, 36 \%$ to $67 \%, \mathrm{I}^{2} 99.4 \%, \mathrm{n}=21$ ). The quality of studies imposed limitations on analyses and the interpretation of results (mean score 3 on a scale of $0-7$ ).

Conclusions: Strengthening of MHM programmes in India is needed. Education on awareness, access to hygienic absorbents and disposal of MHM items need to be addressed.

Trial registration number: CRD42015019197.

\section{Strengths and limitations of this study}

- This study combines current knowledge on menstrual hygiene management (MHM) among adolescents in India.

- The review provides a wide perspective, evaluating multiple outcomes, such as awareness of menarche, source of knowledge on MHM, MHM absorbents used, restrictions during menstruation, and school absenteeism.

- The quality of studies and lack of information on school sanitation imposed limitations on the type of analyses that could be conducted and on the interpretation of results.

- Despite the caveats, our data suggest that such studies can provide data relevant for the development of MHM packages which support girls' menstrual needs.

\section{INTRODUCTION}

Menstrual hygiene management (MHM) is a problem for adolescent girls in low and middle income countries (LMICs), particularly when attending school. ${ }^{1}$ Poor water, sanitation and hygiene (WASH) facilities in schools, inadequate puberty education and lack of hygienic MHM items (absorbents) cause girls to experience menstruation as shameful and uncomfortable (figure 1). ${ }^{2-5}$ Qualitative studies report girls' fear and humiliation from leaking of blood and body odour, and lead menstruating girls to absent themselves from school, ${ }^{2} 67$ with little quantitative data confirming this. ${ }^{7}{ }^{8}$ Cultural taboos add to girls' difficulties, preventing them from seeking help, ${ }^{9} 10$ and impose restrictions on their diet and activities when menstruating. ${ }^{9-12}$ Insufficient $\mathrm{MHM}$ may result in health symptoms involving the urinary and genital tracts. ${ }^{13-15}$ Recent international concern for MHM, spearheaded through work to improve WASH in schools, has focused on the need for dignity and 
Figure 1 Elements important for menstrual hygiene management.

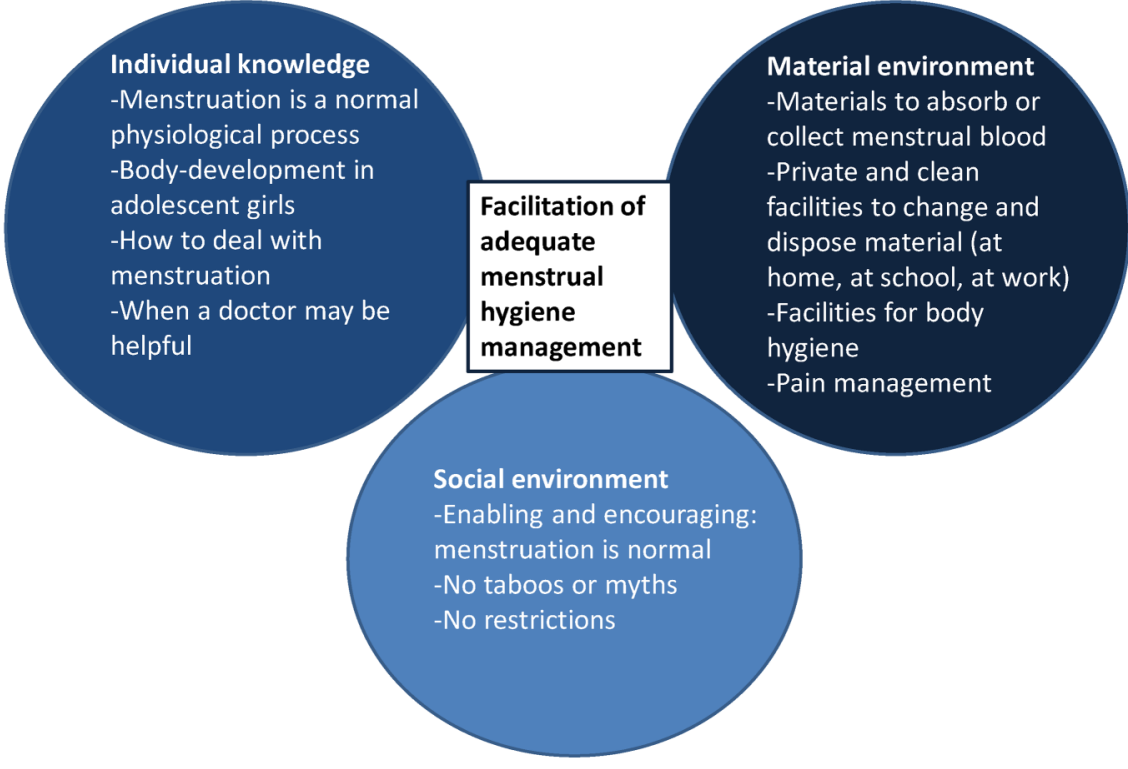

privacy, on raising awareness to break the silence and stigma, making safe and effective MHM absorbents accessible, and improving the school WASH environment. The latter includes separate toilets for girls, water and cleansing materials, and safe disposal of soiled materials. ${ }^{16-19}$

India is a country of contrasts, with extreme wealth and poverty and gender-related disparities, resulting in significant variation in health and social indicators among girls and women. ${ }^{19}$ Of the 113 million adolescent girls, 68 million attend about 1.4 million schools, with poor MHM practices and cultural taboos considered to be impediments to their school attendance. ${ }^{411} 20$ Recognising the relevance of MHM to the health, wellbeing and educational achievements of girls, the Government of India has initiated an array of policies and programmes, implemented at state level. ${ }^{20}$ Numerous MHM studies have been independently conducted across India, examining the prevalence of social, educational and health problems faced by girls with poor MHM. This systematic review and meta-analysis examined all papers published between 2000 and September 2015, to assess the status of MHM among adolescent girls in India to determine unmet and priority needs, and to develop a package of interventions for schoolgirls. The findings can also assist policymakers globally in the development of MHM policies for adolescent girls.

\section{METHODS}

\section{Search strategy and selection criteria}

Methods of analysis and inclusion criteria were specified in advance and documented in the registered Prospero protocol (CRD42015019197). We searched for observational studies with information on MHM (knowledge, attitude, perceptions and practice) among adolescent (10-19 years) girls in India published between 2000 and
September 2015. No language or publication status restrictions were imposed a priori. Studies with mixed age populations were included if adolescent girls were part of the age range, as were studies with mixed designs and studies where MHM was not the main topic. Baseline surveys were extracted from studies with a before-after design. We used PubMed, the Global Health database and Google Scholar using the keywords 'adolescent OR adolescence OR puberty OR peer OR school' AND 'Menstruation OR menstrual OR menses' AND 'hygiene OR hygienically OR sanitation OR sanitary' AND 'India' (see online supplement S1). We searched the 'grey literature' (eg, reports from UNICEF about school sanitation retrieved in Google Scholar), conference abstracts and manually reviewed reference lists of eligible publications. The last search was conducted on 19 September 2015. The following outcomes were examstruation, and sources of the awareness; knowledge of source of bleeding, perception of menstruation as a normal phenomenon, information sources for menstruation, type of absorbent used, disposal of absorbents, daily bathing, restrictions imposed, school absenteeism during menstruation, change of absorbent in school and availability of toilets at home. We use 'absorbent' to refer to any product used to collect menstrual flow; commercial pads refer to those marketed and available for purchase in shops.

\section{Study selection, data extraction}

One reviewer $(\mathrm{AMvE})$ screened abstracts or full texts of all citations obtained for eligibility. Data extraction of eligible material and the quality assessment was conducted independently by two reviewers ( $\mathrm{AMvE}$ and $\mathrm{MS}$ or $\mathrm{AB}$ ) using a data extraction framework (see online supplement S1) and standardised pretested extraction forms. We extracted data on study characteristics (site, year of study, participants, inclusion criteria and sample size) ined: awareness of menarche before the start of men- 
and outcomes (number and total). Differences were resolved by discussion among co-authors. The abstracted data were entered into a database for analysis. We did not contact authors for further information. No systematic data extraction or analyses were conducted for qualitative components present in included studies with mixed designs (table 1).

\section{Study quality}

Ad hoc quality assessments methods are detailed in online supplement S1. Quality assessments of included studies were conducted by the same reviewers on the basis of the following seven criteria: presence of a sample size calculation, randomness of sample, description and appropriateness of inclusion and exclusion criteria, presence of a description of the number of persons approached but not enrolled, or enrolled but data not used, completeness of outcome data for the number which was presented, presence of participant characteristics and of multivariate analysis for the outcome of the paper. The results were added to obtain an overall quality score ranging from 0 to 7 for each study. This quality score was not based on a preexisting instrument, and has not been validated in other studies. Studies were not excluded on the basis of their quality.

\section{Data synthesis}

Prevalence was pooled as a proportion in a random-effects model ('metaprop', Stata V.13, StataCorp LP, College Station, USA) using the Freeman-Tukey double arcsine transformation and computation of the CI using the 'exact method'. ${ }^{21}{ }^{22}$ The extent of heterogeneity was measured using the $\mathrm{I}^{2}$, a measure of the proportion of total variability explained by heterogeneity rather than chance expressed as a percentage. ${ }^{23}$ To explore determinants of prevalence, we examined sources of heterogeneity across studies of the pooled prevalence (PP) estimates using random-effects meta-regression. $^{24}$ Regression coefficients were presented as ORs with corresponding 95\% CIs. We estimated between-study variance $\left(\tau^{2}\right)$ using the algorithm of residual (restricted) maximum likelihood and calculated $p$ values, and 95\% CI for coefficients using the modification by Knapp and Hartung. ${ }^{25}$

Study-level predictors were included in the initial models if the $\mathrm{p}$ value for the univariate association was $<0.2$. Backwards elimination was conducted whereby variables were removed if the $p$ value was $>0.1$ in the multivariate model (see online supplement 1 , section S1.4). We examined the effect of study location (urban, rural, slum or combined), year of study, source of study population (community, school or other), region (North, Central, East, West and South), and if the study population was completely or only partially adolescents. For sensitivity analysis, we examined the effect of selfadministered questionnaire versus study staff interview, study quality assessment as a continuous or dichotomous
Table 1 Characteristics of included Indian studies on Menstrual Hygiene Management published between 2000 and 2015

\begin{tabular}{|c|c|c|}
\hline Variable & Values & $\begin{array}{l}\text { Number (\%) } \\
\mathrm{N}=138\end{array}$ \\
\hline \multirow[t]{3}{*}{ Type of material } & Article & $127(92.0)$ \\
\hline & Report & $9(6.5)$ \\
\hline & Thesis & $2(1.5)$ \\
\hline \multirow[t]{5}{*}{ Year of publication } & 2000 & $1(0.7)$ \\
\hline & 2001-2004 & $7(5.1)$ \\
\hline & 2005-2009 & $24(17.4)$ \\
\hline & 2010-2014 & 90 (65.2) \\
\hline & 2015 & 16 (11.6) \\
\hline \multirow[t]{3}{*}{ Study design } & Survey & 107 (77.5) \\
\hline & Before/after design* & 19 (13.8) \\
\hline & Mixed methods & $12(8.7)$ \\
\hline \multirow[t]{2}{*}{ Study population } & $\begin{array}{l}\text { Completely } \\
\text { adolescent girls }\end{array}$ & $118(85.5)$ \\
\hline & $\begin{array}{l}\text { Partly adolescent } \\
\text { girls }\end{array}$ & $20(14.5)$ \\
\hline \multirow[t]{4}{*}{ Setting } & Rural & $50(36.2)$ \\
\hline & Urban & $48(34.8)$ \\
\hline & Urban and rural & $30(21.7)$ \\
\hline & Slum & $10(7.3)$ \\
\hline \multirow{3}{*}{$\begin{array}{l}\text { Location of } \\
\text { recruitment }\end{array}$} & School & $82(59.4)$ \\
\hline & Community & $45(32.6)$ \\
\hline & Other† & $11(8.0)$ \\
\hline \multirow{3}{*}{$\begin{array}{l}\text { Method of data } \\
\text { collection }\end{array}$} & Self-administered & $69(50.0)$ \\
\hline & $\begin{array}{l}\text { Interview by study } \\
\text { staff }\end{array}$ & 68 (49.3) \\
\hline & Not applicable & $1 \ddagger(0.7)$ \\
\hline \multirow{4}{*}{$\begin{array}{l}\text { Time period of } \\
\text { study§ }\end{array}$} & Before 2000 & $5(3.6)$ \\
\hline & 2000-2004 & 17 (12.3) \\
\hline & 2005-2009 & $35(25.4)$ \\
\hline & 2010-2014 & $81(58.7)$ \\
\hline \multirow[t]{5}{*}{ Regionๆ } & North & $27(19.7)$ \\
\hline & Central & $11(8.0)$ \\
\hline & East & 19 (13.9) \\
\hline & West & $32(23.4)$ \\
\hline & South & 48 (35.0) \\
\hline \multicolumn{2}{|l|}{$\begin{array}{l}\text { Median sample } \\
\text { size (range) }\end{array}$} & $322(30-5000)$ \\
\hline \multicolumn{3}{|c|}{$\begin{array}{l}{ }^{*} \text { Two studies with a before/after design had a mixed design } \\
\text { (quantitative and qualitative components). } \\
\text { +Clinic } 4 \text {, Hostel 2, Vocational training centre 2, School and } \\
\text { community 2, not reported } 1 . \\
\text { łln this study, girls were given the opportunity to ask questions on } \\
\text { MHM, and the contents of questions were analysed. } \\
\S \text { Estimated for } 59 \text { studies where this was not reported by using } \\
\text { the median between last study year and publication year (2 years) } \\
\text { for studies where the time of the study was reported (79 studies). } \\
\text { INorth: New Delhi, Haryana, Jammu and Kashmir, Punjab, } \\
\text { Rajasthan, Uttarakhand; Central: Chhattisgarh, Madhya Pradesh, } \\
\text { Uttar Pradesh; East: Bihar, Jharkhand, Orissa, West Bengal, } \\
\text { Meghalaya; West: Gujarat, Maharashtra; South: Andhra Pradesh, } \\
\text { Karnataka, Kerala, Tamil Nadu, Puducherry, Telangana. One } \\
\text { study had a national sample (Anand 2015) and was not included } \\
\text { here. }{ }^{15} \\
\text { MHM, Menstrual Hygiene Management. }\end{array}$} \\
\hline
\end{tabular}

variable (quality score $>4$ vs $\leq 4$ ), and the effect of random sampling using meta-regression. Quality scores were not used as weights in analyses. 


\section{RESULTS}

\section{Characteristics and quality}

We retrieved 145 studies through the database searches, and another 80 potentially eligible studies through reference lists. After exclusion and de-duplication, 138 were left (see online supplementary table S1.6). Most studies were in Maharashtra (24 studies; $17.4 \%$ ) with a surge since 2010 (table 1 and figure 2). Studies were mostly of low-to-moderate quality (see online supplementary table S1.4 and S1.5). Frequently missing information was the number of refusals or exclusions; multivariate analyses were usually missing $(90 \%)$. The sample size varied from small to very large (range $30-38928$, total 97070 ), often without sample calculations $(71.0 \%)$. In total, $19.6 \%$ of studies had five or more quality criteria fulfilled.

\section{Premenarche awareness and sources of information}

Among 88 studies with available information, the pooled prevalence of premenarche awareness was $48 \%$ (95\% CI $43 \%$ to $53 \%, \mathrm{I}^{2} 98 \%$, range $3-100 \%$ ). In multivariate analysis, the prevalence was affected by setting, region and year of study with significantly higher awareness over time, and in the East and West of India compared to the North, and lower awareness in slum settings (see online supplementary table S2.2). Among girls aware prior to menarche, mothers were the most frequent source of information (PP 52\%, 47\% to 57\%, I ${ }^{2} 94 \%$, $\mathrm{n}=43$ ), followed by friends (PP $27 \%, 22 \%$ to $32 \%, \mathrm{I}^{2}$ $95 \%, \mathrm{n}=37$ studies). Other sources such as relatives (including sisters) and teachers were less commonly reported (table 2) and there were some regional differences (see online supplementary table S2.3).

\section{Knowledge and perception of menstruation and knowledge resources}

Overall, about a quarter of girls knew that the uterus is the source of bleeding (PP 23\%, 16\% to 30\%, range 1$97 \%, \mathrm{I}^{2} 99 \%$ ), and approximately half considered menstruation normal (PP 55\%, 47\% to 63\%, range 6-98\%, $\mathrm{I}^{2} 99 \%$, table 2). Mothers were most commonly mentioned as the knowledge source in 41 studies, with half of the girls reporting her as the main source (PP 54\%,

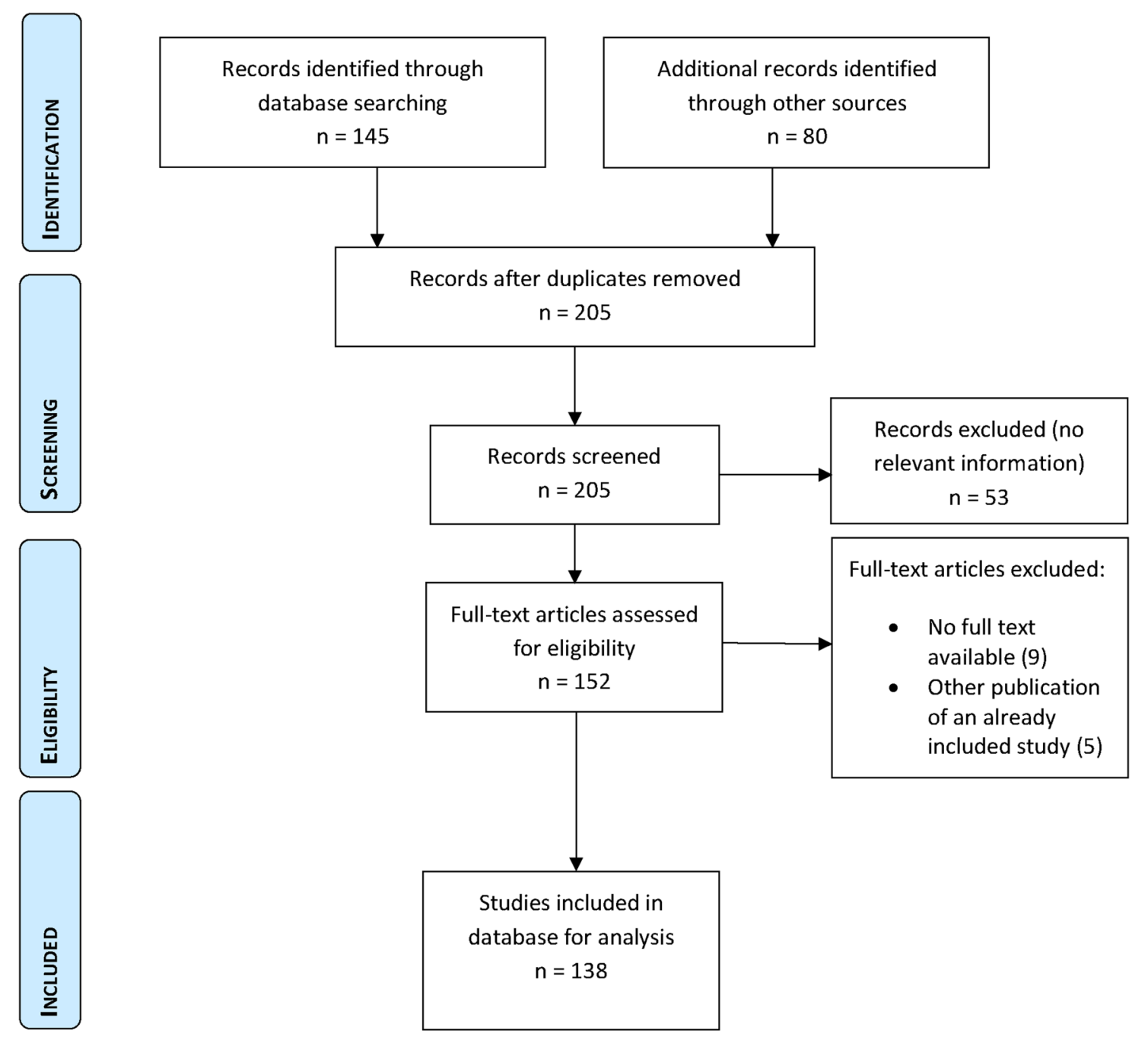

Figure 2 Flow chart of systematic review of MHM studies among adolescent girls in India. MHM, Menstrual Hygiene Management. 
Table 2 Pooled proportions of awareness of menarche, knowledge of menstruation, and sources of information about menarche and menstruation, overall and by setting, in Indian studies published between 2000 and 2015

\begin{tabular}{|c|c|c|c|c|c|c|c|c|c|c|}
\hline \multirow[b]{2}{*}{ Factor* } & \multicolumn{2}{|c|}{ Total pooled } & \multicolumn{2}{|c|}{ Rural } & \multicolumn{2}{|c|}{ Urban } & \multicolumn{2}{|c|}{ Slum } & \multicolumn{2}{|c|}{ Combination } \\
\hline & $\mathbf{N}$ & Proportion, 95\% Cl & $\mathbf{N}$ & Proportion, 95\% Cl & $\mathbf{N}$ & Proportion, 95\% Cl & $\mathbf{N}$ & Proportion, 95\% Cl & $\overline{\mathbf{N}}$ & Proportion, $95 \% \mathrm{Cl}$ \\
\hline Aware of menarche & 88 & $0.48,0.43$ to 0.53 & 39 & $0.45,0.38$ to 0.51 & 37 & $0.53,0.44$ to 0.62 & 5 & $0.26,0.20$ to 0.33 & 7 & $0.60,0.52$ to 0.68 \\
\hline \multicolumn{11}{|c|}{ Information source on menarche among girls aware of menarche } \\
\hline Mother & 43 & $0.52,0.47$ to 0.57 & 15 & $0.52,0.43$ to 0.61 & 18 & $0.50,0.41$ to 0.60 & 4 & $0.59,0.52$ to 0.65 & 5 & $0.55,0.47$ to 0.63 \\
\hline Friends & 37 & $0.27,0.22$ to 0.32 & 12 & $0.32,0.23$ to 0.42 & 15 & $0.24,0.17$ to 0.32 & 3 & $0.20,0.14$ to 0.26 & 5 & $0.26,0.16$ to 0.37 \\
\hline Relative & 26 & $0.18,0.15$ to 0.21 & 10 & $0.15,0.09$ to 0.23 & 14 & $0.19,0.14$ to 0.25 & 4 & $0.15,0.03$ to 0.31 & 4 & $0.23,0.17$ to 0.28 \\
\hline Teacher & 25 & $0.12,0.07$ to 0.17 & 8 & $0.11,0.05$ to 0.20 & 12 & $0.13,0.05$ to 0.23 & 2 & $0.15,0.09$ to 0.21 & 3 & $0.07,0.03$ to 0.11 \\
\hline Mediał & 17 & $0.15,0.09$ to 0.22 & 6 & $0.17,0.06$ to 0.31 & 9 & $0.16,0.06$ to 0.28 & 0 & & 2 & $0.08,0.00$ to 0.25 \\
\hline Health worker & 4 & $0.18,0.00$ to 0.63 & 1 & $0.01,0.00$ to 0.06 & 2 & $0.42,0.00$ to 1.00 & 1 & $0.06,0.01$ to 0.19 & 0 & \\
\hline \multicolumn{11}{|c|}{ Knowledge on menstruation and perception } \\
\hline Uterus is a source of bleeding & 43 & $0.23,0.16$ to 0.30 & 26 & $0.19,0.12$ to 0.27 & 11 & $0.35,0.18$ to 0.54 & 3 & $0.17,0.06$ to 0.31 & 3 & $0.23,0.03$ to 0.52 \\
\hline $\begin{array}{l}\text { Menstruation is a normal } \\
\text { phenomenon }\end{array}$ & 54 & $0.55,0.47$ to 0.63 & 25 & $0.53,0.39$ to 0.66 & 23 & $0.63,0.51$ to 0.73 & 4 & $0.43,0.30$ to 0.56 & 2 & $0.25,0.13$ to 0.38 \\
\hline \multicolumn{11}{|c|}{ Resources on menstruation among participants } \\
\hline Mother & 41 & $0.54,0.45$ to 0.63 & 17 & $0.53,0.41$ to 0.64 & 17 & $0.58,0.44$ to 0.70 & 4 & $0.49,0.10$ to 0.90 & 3 & $0.45,0.05$ to 0.90 \\
\hline Friend & 35 & $0.24,0.18$ to 0.30 & 16 & $0.25,0.19$ to 0.32 & 15 & $0.18,0.11$ to 0.26 & 2 & $0.24,0.00$ to 0.77 & 2 & $0.56,0.07$ to 0.98 \\
\hline Relative $†$ & 31 & $0.14,0.10$ to 0.19 & 13 & $0.14,0.10$ to 0.19 & 13 & $0.16,0.08$ to 0.25 & 2 & $0.24,0.11$ to 0.40 & 3 & $0.06,0.02$ to 0.13 \\
\hline Teacher & 23 & $0.08,0.05$ to 0.11 & 10 & $0.11,0.05$ to 0.19 & 9 & $0.05,0.03$ to 0.08 & 2 & $0.10,0.08$ to 0.12 & 2 & $0.04,0.02$ to 0.07 \\
\hline Mediał & 24 & $0.10,0.06$ to 0.15 & 11 & $0.07,0.03$ to 0.11 & 10 & $0.14,0.07$ to 0.22 & 1 & $0.33,0.30$ to 0.37 & 2 & $0.08,0.00$ to 0.30 \\
\hline Health worker & 8 & $0.08,0.05$ to 0.12 & 5 & $0.09,0.05$ to 0.15 & 2 & $0.08,0.05$ to 0.13 & 1 & $0.04,0.02$ to 0.08 & 0 & \\
\hline
\end{tabular}

${ }^{*}$ Other subgroup analyses and meta-regression for factors in this table: See online supplement S2, tables S2.2 and S2.3. For forest plots, see online supplement S3.

Sisters or other relative.

Books, movies, radio, magazines and internet. 
$45 \%$ to $63 \%, \mathrm{I}^{2} 99 \%$, table 2). Friends and relatives were regularly mentioned with a PP of $24 \%$ and $14 \%$, respectively, whereas teachers, media and health workers were uncommon resources with PPs of $\leq 10 \%$ (table 2).

\section{Type of menstrual absorbents used}

The use of absorbents was strikingly different by setting, with commercial pads significantly more common in urban areas, and cloths significantly more common in rural areas (table 3). Commercial pad use increased significantly over time $(p<0.0001$, online supplementary table S2.4 and figure 3). Cloth use was less common in school studies (PP 37\%, 95\% CI 29\% to $45 \%$, I ${ }^{2} 99 \%$, $\mathrm{n}=57$ ) compared with community studies (PP 68, 95\% CI $59 \%$ to $76 \%, \mathrm{I}^{2} 99 \%, \mathrm{n}=38$ ), independent of setting (meta-regression: $\mathrm{p}=0.007$ for school vs community recruitment and $\mathrm{p}<0.001$ for urban vs rural setting, online supplementary table S2.5). Some girls combined pad and cloth use, with a PP of $13 \%$ (95\% CI 9\% to $\left.18 \%, \mathrm{I}^{2} 99 \%, \mathrm{n}=42\right)$; this was higher among school studies $\left(18 \%, 95 \%\right.$ CI $13 \%$ to $\left.24 \%, \mathrm{I}^{2} 98 \%, \mathrm{n}=15\right)$ compared with community studies (PP 6\%, 95\% CI 2\% to $11 \%, \mathrm{I}^{2} 98 \%, \mathrm{n}=26, \mathrm{p}<0.001$, online supplementary table S2.5). One study in urban Tamil Nadu asked about materials inserted into the vagina (reported by $26.9 \%$ ), but it was unclear if these were tampons. ${ }^{26}$ Only one study (in urban Karnataka) mentioned tampons, with five girls reportedly using them. ${ }^{27}$ No studies reported menstrual cups. Few studies mentioned contraceptives to postpone menstruation (ie, to attend events without menstrual discomfort). ${ }^{28} \quad 29$ Economic considerations seemed to be the main reason for using cloths instead of pads; other reasons included difficulty with disposal, unawareness of pads, or personal preference (see online supplementary table S2.6).

\section{Disposal of absorbents}

Disposal in urban settings was mainly through routine waste and burning (table 3), while in rural settings burying and throwing away in public spaces was also common. Routine waste disposal was significantly more frequent in urban (PP 70\%, 60\% to $79 \%, \mathrm{I}^{2} 98 \%, \mathrm{n}=16$ ) compared to rural settings (PP 28\%, 95\% CI 19\% to $38 \%, \mathrm{I}^{2} 99 \%, \mathrm{n}=25, \mathrm{p}<0.0001$, online supplementary table S2.5). Burying was more common in communitybased studies $\left(46 \%, 34 \%\right.$ to $\left.57 \%, \mathrm{I}^{2} 99 \%, \mathrm{n}=10\right)$ than school-based studies (PP $14 \%, 9 \%$ to $20 \%, \mathrm{I}^{2} 97 \%, \mathrm{n}=13$, $\mathrm{p}=0.003$, online supplementary table S2.5). About $23 \%$ of girls threw the absorbent in open spaces, such as the roadside or in rivers and lakes; this was significantly higher in community-based studies (PP 35\%, 24\% to $\left.46 \%, \mathrm{I}^{2} 99 \%, \mathrm{n}=14\right)$ than in school-based studies $(16 \%$, $8 \%$ to $26 \%, \mathrm{I}^{2} 99 \%, \mathrm{n}=18, \mathrm{p}=0.001$, online supplementary table S2.5).

\section{Hygiene during menstruation}

Most participants $(84 \%, 95 \%$ CI $76 \%$ to $91 \%$, range $2-$ $99 \%, \mathrm{I}^{2} 99 \%, \mathrm{n}=39$ ) bathed daily during menstruation, with no difference between urban and rural settings; bathing was significantly less in one study in a slum (table 3). A subgroup of rural studies found that daily baths were less common during versus before or after menstruation. ${ }^{30-32}$ Other studies also noted bathing restrictions for girls during menstruation, due to fear that bathing might cause problems in future pregnancies, a scarcity of water, non-availability of bathing space, physical discomfort and lack of privacy. ${ }^{10} 28$ 33-36

\section{Restrictions}

Restrictions during menstruation are common. The most frequent were restrictions in visiting places of worship, and touching religious items or praying (PP $77 \%, 71 \%$ to $83 \%$, range $7-100, \mathrm{I}^{2} 99 \%, \mathrm{n}=67$ ), with no difference by setting (table 4) or over time (figure 4). Other common restrictions were food related (PP 38\%, $30 \%$ to $\left.46 \%, \mathrm{I}^{2} 99 \%, \mathrm{n}=44\right)$, or touching people or special foods (PP 50\%, 36\% to $64 \%, \mathrm{I}^{2} 99 \%, \mathrm{n}=19$ ). In some studies, girls reported sleeping separately (PP $26 \%, 14 \%$ to $39 \%, \mathrm{I}^{2} 99 \%, \mathrm{n}=20$ ) or sitting separately from household members during menstruation (PP $24 \%$, $7 \%$ to $46 \%, \mathrm{I}^{2} 98 \%, \mathrm{n}=6$ ). Girls faced restrictions in cooking, household work, exercise and playing, moving in and out of the house, and attending social functions (see table 4, online supplementary table S2.7 and figure S2.1 for a list of other reported beliefs and restrictions).

\section{School absenteeism}

Sixty-four studies reported on school absenteeism associated with menstruation, with one in four girls missing one or more school days during menstruation (PP 24\%, $19 \%$ to $30 \%, \mathrm{I}^{2} 99 \%$, table 5 ), with significant differences across regions (figure 5). Girls' reasons for absence were physical discomfort or pain, lack of water, hygiene and disposal facilities in school toilets, fear of staining their clothes and restrictions imposed by relatives or teachers (see online supplementary table S2.8). No trend over time was seen $(\mathrm{p}=0.945$, online supplementary table S2.9). In a univariate model, commercial pad use as a continuous variable was associated with a decrease in absenteeism ( $\mathrm{p}=0.023, \mathrm{n}=53$, figure 6$)$, but not in a multivariate model when adjusted for region $(\mathrm{p}=0.232)$. Similarly, cloth use was associated with an increase in absenteeism in a univariate $(\mathrm{p}=0.042, \mathrm{n}=48)$ but not in a multivariate model $(\mathrm{p}=0.543)$. An average of just over a third (PP 37\%, 29\% to $46 \%, \mathrm{I}^{2} 98 \%, \mathrm{n}=17$ ) of girls reported changing their absorbent in school while others avoided this (table 5 ). Toilets at home were present in about half (PP $51 \%, 95 \%$ CI $36 \%$ to $67 \%, \mathrm{I}^{2}$ $99 \%, \mathrm{n}=21$ ) of the homes of the girls involved, with a significant difference between urban and rural settings (table 5).

\section{Sensitivity analyses}

For several outcomes, the quality score or method of data collection made a difference, and was generally 
Table 3 Pooled proportions of absorbents used, the disposal of the absorbent and use of a daily bath during menstruation, in Indian studies published between 2000 and 2015

\begin{tabular}{|c|c|c|c|c|c|c|c|c|c|c|}
\hline \multirow[b]{2}{*}{ Factor* } & \multicolumn{2}{|c|}{ Total pooled } & \multicolumn{2}{|c|}{ Rural } & \multicolumn{2}{|c|}{ Urban } & \multicolumn{2}{|c|}{ Slum } & \multicolumn{2}{|c|}{ Combination } \\
\hline & $\mathbf{N}$ & $\begin{array}{l}\text { Pooled proportion, } \\
95 \% \mathrm{Cl}\end{array}$ & $\mathbf{N}$ & $\begin{array}{l}\text { Pooled proportion, } \\
95 \% \mathrm{Cl}\end{array}$ & $\mathbf{N}$ & $\begin{array}{l}\text { Pooled proportion, } \\
95 \% \mathrm{Cl}\end{array}$ & $\mathbf{N}$ & $\begin{array}{l}\text { Pooled proportion, } \\
95 \% \mathrm{Cl}\end{array}$ & $\mathbf{N}$ & $\begin{array}{l}\text { Pooled proportion, } \\
95 \% \mathrm{Cl}\end{array}$ \\
\hline \multicolumn{11}{|l|}{ Absorbents used } \\
\hline Commercial pads & 105 & $0.45,0.38$ to 0.52 & 56 & $0.32,0.25$ to 0.38 & 38 & $0.67,0.57$ to $0.76 \dagger$ & 9 & $0.43,0.20$ to 0.67 & 2 & $0.34,0.00$ to 0.87 \\
\hline Cloths & 102 & $0.50,0.43$ to 0.58 & 55 & $0.63,0.56$ to 0.70 & 35 & $0.27,0.18$ to $0.37 \dagger$ & 8 & $0.61,0.35$ to 0.83 & 4 & $0.73,0.41$ to 0.95 \\
\hline Pads and cloths & 42 & $0.13,0.09$ to 0.18 & 23 & $0.09,0.05$ to 0.13 & 14 & $0.21,0.13$ to $0.31 \dagger$ & 4 & $0.10,0.06$ to 0.16 & 1 & $0.25,0.16$ to 0.36 \\
\hline Cottons/home-made disposables & 12 & $0.04,0.02$ to 0.06 & 8 & $0.04,0.02$ to 0.07 & 4 & $0.04,0.01$ to 0.08 & 0 & & 0 & \\
\hline Underwear only & 7 & $0.06,0.01$ to 0.14 & 6 & $0.07,0.01$ to 0.17 & 1 & $0.02,0.01$ to 0.05 & & & & \\
\hline \multicolumn{11}{|l|}{ Disposal of absorbent } \\
\hline Using routine waste/dustbin & 44 & $0.45,0.35$ to 0.55 & 25 & $0.28,0.19$ to 0.38 & 16 & $0.70,0.60$ to $0.79 \dagger$ & 3 & $0.51,0.47$ to 0.55 & 0 & \\
\hline ‘Throw away’ & 34 & $0.23,0.16$ to 0.31 & 19 & $0.28,0.19$ to 0.38 & 13 & $0.15,0.09$ to 0.24 & 2 & $0.30,0.04$ to 0.66 & 0 & \\
\hline Burning & 31 & $0.17,0.08$ to 0.29 & 22 & $0.15,0.07$ to 0.24 & 9 & $0.23,0.05$ to 0.49 & 0 & & 0 & \\
\hline Burying & 24 & $0.25,0.16$ to 0.35 & 16 & $0.33,0.22$ to 0.45 & 8 & $0.12,0.06$ to $0.19 \dagger$ & 0 & & 0 & \\
\hline In toilet§ & 17 & $0.09,0.04$ to 0.17 & 7 & $0.10,0.00$ to 0.30 & 10 & $0.07,0.03$ to 0.12 & 0 & & 0 & \\
\hline \multicolumn{11}{|l|}{ Hygiene } \\
\hline Daily bath during menstruation & 39 & $0.84,0.76$ to 0.91 & 24 & $0.86,0.76$ to 0.93 & 12 & $0.92,0.86$ to 0.97 & 1 & $0.15,0.13$ to $0.18 \dagger$ & 2 & $0.33,0.00$ to $1.00 \dagger$ \\
\hline
\end{tabular}




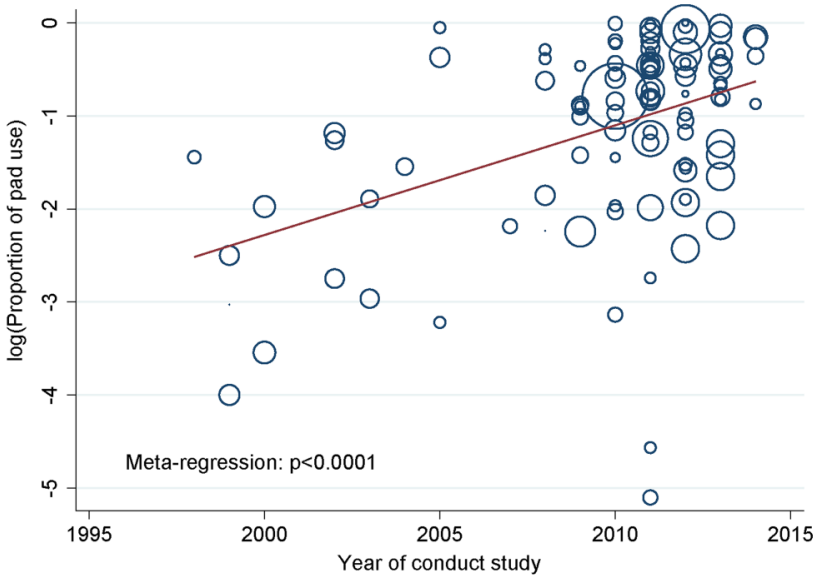

Figure 3 Bubble plot with a fitted meta-regression line of the log prevalence of commercial pad use by adolescent girls over time, 104 studies in India published between 2000 and 2015. Circles are sized according to the precision of each estimate with larger bubbles for more precise estimates. One study was excluded ${ }^{15}$ because of the large sample size $(\approx 39000)$, which led to distortion of all other information.

associated with a decrease in prevalence, for example, a higher quality score of a study with a lower awareness of menarche prior to it (see online supplementary table S2.2), and lower use of commercial pads (see online supplementary table S2.4); a random sample was associated with a lower prevalence of 'throwing away' the absorbent (see online supplementary table S2.5). A selfadministered interview was associated with a lower prevalence of the use of media as a resource for knowledge on menstruation (see online supplementary table S2.3), and a lower prevalence of absenteeism (online supplementary table S2.9).

\section{DISCUSSION}

On the basis of this review, we estimate that about half of Indian adolescent girls started menarche unaware of its cause, with only a quarter understanding the source of bleeding. The majority of girls faced numerous barriers and restrictions; only one in eight girls faced no restriction at all. Commercial pads were more commonly used in urban settings or schools, with girls in rural areas and in community-based studies mainly dependent on cloths. About one in five girls disposed their soiled absorbents in inappropriate locations. A quarter of girls reported that they did not attend school during menstruation. Absenteeism due to menstruation did not decrease over time; school absence was inversely associated with the prevalence of pad use in univariate analysis, but not when adjusted for region.

Cloths are traditionally used to absorb menstrual flow; they are cheaper and environmentally less polluting, but are gradually being replaced by pads, particularly in urban areas. Cleaning and drying cloths is a problem if girls lack water, privacy and a drying place. ${ }^{10} 37$ Drying

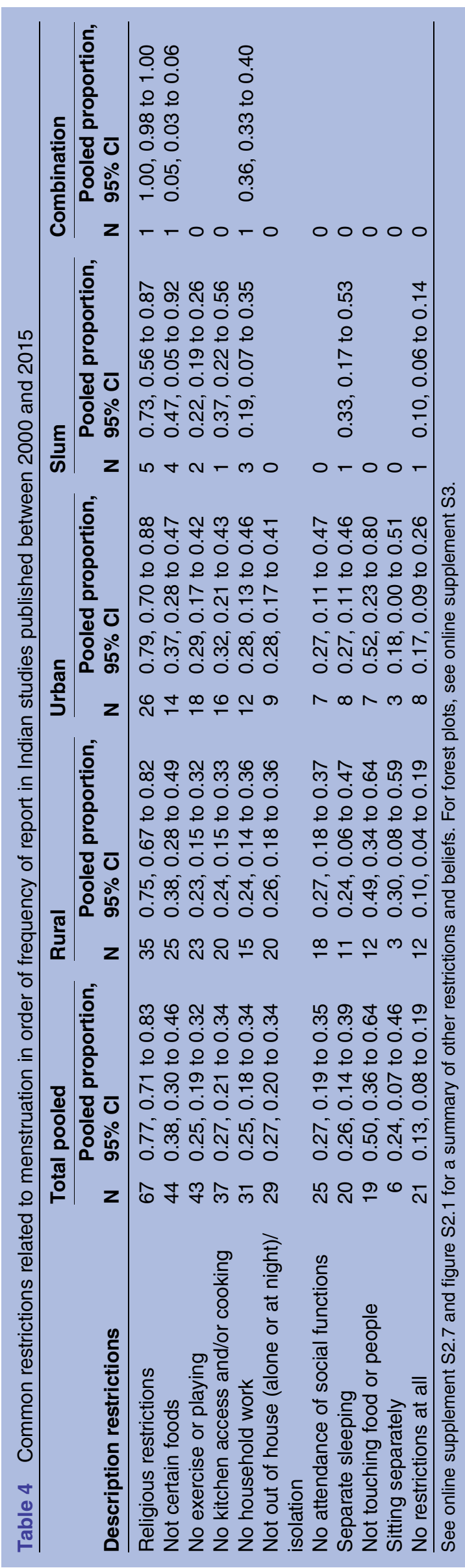



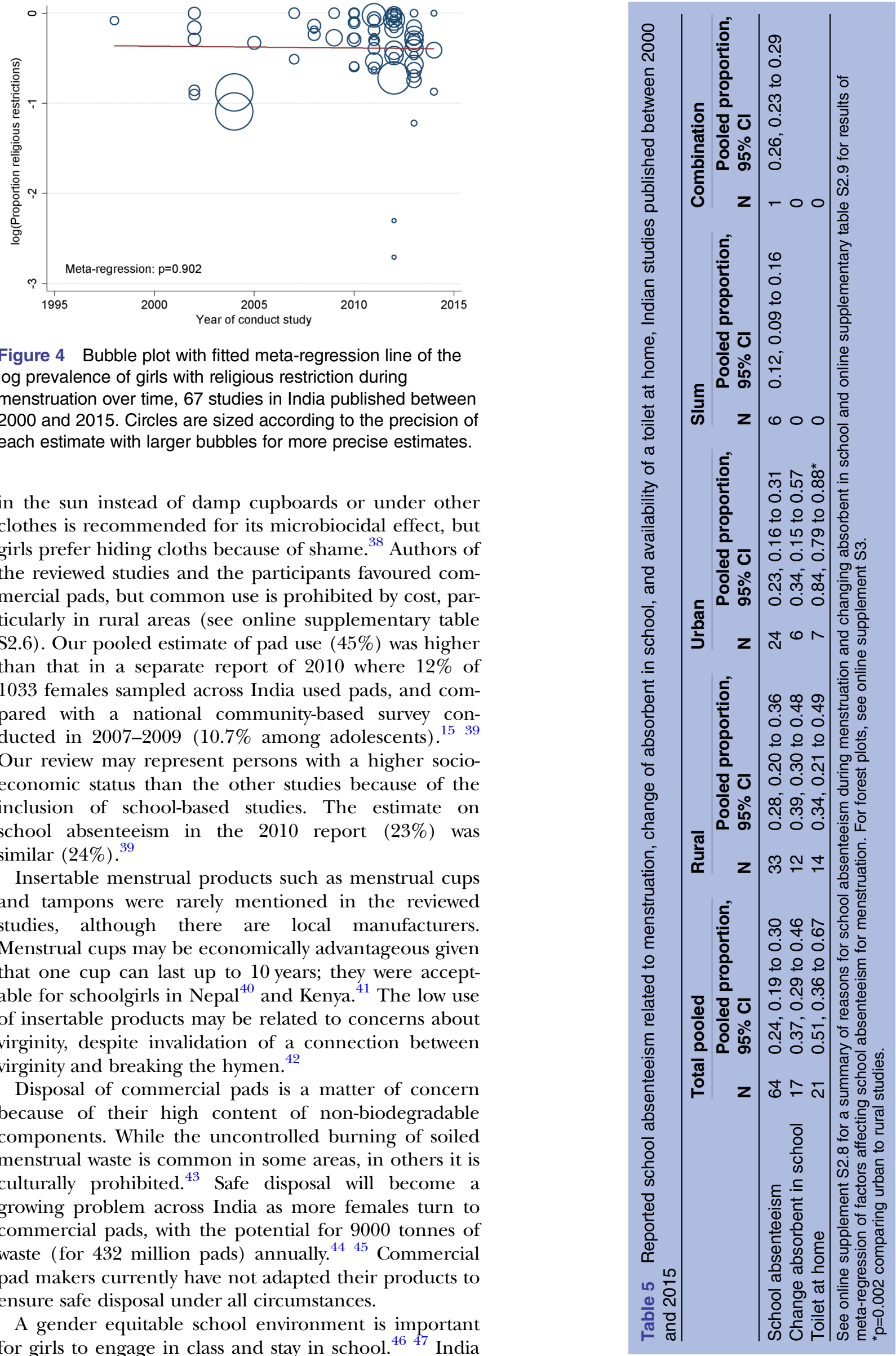

Figure 4 Bubble plot with fitted meta-regression line of the log prevalence of girls with religious restriction during menstruation over time, 67 studies in India published between 2000 and 2015. Circles are sized according to the precision of each estimate with larger bubbles for more precise estimates.

in the sun instead of damp cupboards or under other clothes is recommended for its microbiocidal effect, but girls prefer hiding cloths because of shame. ${ }^{38}$ Authors of the reviewed studies and the participants favoured commercial pads, but common use is prohibited by cost, particularly in rural areas (see online supplementary table S2.6). Our pooled estimate of pad use (45\%) was higher than that in a separate report of 2010 where $12 \%$ of 1033 females sampled across India used pads, and compared with a national community-based survey conducted in 2007-2009 (10.7\% among adolescents). ${ }^{15} 39$ Our review may represent persons with a higher socioeconomic status than the other studies because of the inclusion of school-based studies. The estimate on school absenteeism in the 2010 report (23\%) was similar $(24 \%){ }^{39}$

Insertable menstrual products such as menstrual cups and tampons were rarely mentioned in the reviewed studies, although there are local manufacturers. Menstrual cups may be economically advantageous given that one cup can last up to 10 years; they were acceptable for schoolgirls in Nepal ${ }^{40}$ and Kenya. ${ }^{41}$ The low use of insertable products may be related to concerns about virginity, despite invalidation of a connection between virginity and breaking the hymen. ${ }^{42}$

Disposal of commercial pads is a matter of concern because of their high content of non-biodegradable components. While the uncontrolled burning of soiled menstrual waste is common in some areas, in others it is culturally prohibited. ${ }^{43}$ Safe disposal will become a growing problem across India as more females turn to commercial pads, with the potential for 9000 tonnes of waste (for 432 million pads) annually. ${ }^{44}$ Commercial pad makers currently have not adapted their products to ensure safe disposal under all circumstances.

A gender equitable school environment is important for girls to engage in class and stay in school. ${ }^{46}$ India 


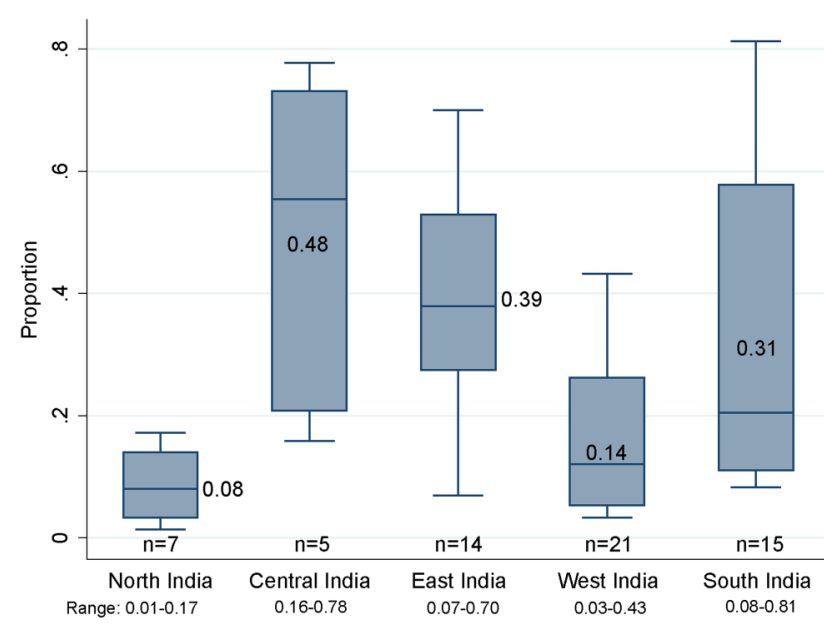

Figure 5 Tukey box plot of school absenteeism reported by adolescent girls during menstruation by region, 64 studies in India published between 2000 and 2015. The bottom and top of the box are the 25th centile and the 75th centile, and the band inside the box is the median. The lower whisker indicates the data between the 25th centile minus 1.5 times the difference between the 25th and 75th centiles, and the upper whisker indicates the data between the 75th centile plus 1.5 times the difference between the 25th and 75th centiles. The mean is indicated next or in the box plot, and the range of values is indicated under each region.

has an array of policies and schemes developed to provide pads, counselling from frontline workers, and the construction of toilets for girls. ${ }^{20}$ The number of separate and usable toilet facilities for girls has increased in India. ${ }^{48}$ Qualitative studies report that school absenteeism is associated with poor MHM interventions, ${ }^{50} 51$ but so far only WASH studies have shown an association between toilet improvement and absenteeism, ${ }^{52} 53$ and improved enrolment of adolescent girls when girls-only toilets were constructed. ${ }^{6}$ We detected a

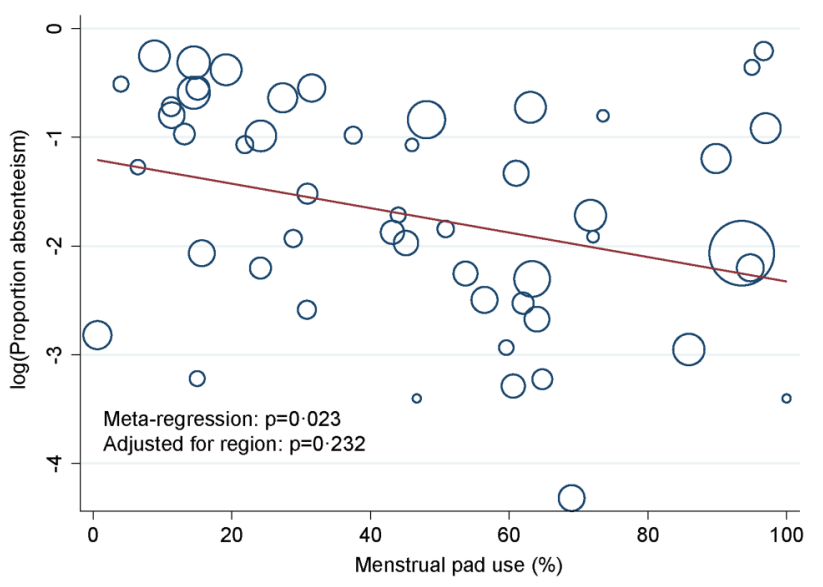

Figure 6 Bubble plot with fitted meta-regression line of the log prevalence of school absenteeism among adolescent girls and menstrual pad use, 53 studies in India published between 2000 and 2015. Circles are sized according to the precision of each estimate with larger bubbles for more precise estimates. relationship between pad use and girls' absenteeism in univariate analysis, but not when adjusted for region. This relationship may be confounded by socioeconomic status and WASH, as schools with a high prevalence of commercial pad use may also be more resourceful with investments for school toilets and cleaning. In our review, only one-third of girls reported using school toilets to change their menstrual absorbent. It is noted that inadequate toilets are not the only reason for menstrual-related absenteeism; physical discomfort was also frequently mentioned (see online supplementary table S2.10). The lack of toilets at home is of great concern with regard to safety, privacy and dignity, as reported repeatedly by women in India. ${ }^{54-56}$

This study has several limitations. There was high heterogeneity between studies as indicated by the $\mathrm{I}^{2}$ statistic, which was regularly over $90 \%$. This may be explained by the different methodology of the studies and the different settings where the studies were conducted. We used meta-regression to explore factors that affected the outcomes, and indeed the setting (urban or rural), region, time and quality of the study were regularly associated with the outcomes examined (see online supplement S2). ${ }^{57}$ Not all studies reached scientific rigour, and states in the Central and Eastern region are under-represented as few studies have been conducted in these regions. To evaluate the potential effect of study quality, we assessed outcomes against the quality score and presence of random samples. Some outcomes were affected, for example, a higher quality score of a study was associated with a lower use of commercial pads and the pooled estimate may have overestimated the prevalence of commercial pad use. Studies were conducted by selfadministered questionnaires or interviews by study staff; the latter may have led to a desirability effect, more so taking the age of respondents into account. A selfadministered questionnaire was associated with a lower prevalence of absenteeism, so the pooled estimate for absenteeism may be overestimated. Studies were crosssectional, so causality cannot be inferred. Further, our review was limited by the information available; not all included studies focused solely on MHM and not all studies presented basic information on age or socioeconomic status. This limited exploration of the effect of factors such as socioeconomic status, caste or maternal education on the outcomes. We aggregated the variety of responses around sources on menarche or menstruation and restrictions faced by girls where possible, and some may have been misclassified. Absence of school during menstruation was often not well defined. The quality of information on the reaction of girls to menarche or how a girl cleans herself during her period, the frequency of changing menstrual materials, and the obstructions girls face was generally too poor to use.

Although menarche is celebrated in some regions of India, ${ }^{10} 33$ 58-60 our findings highlight that for the majority of girls, 'menstruation poses a huge physical and psychological burden'. 33 Societal beliefs and restrictions 
add to these negative experiences. While education could dispel some restrictions (eg, of foods or activities), beliefs and negative attitudes are deeply entrenched within Hindu and other religions. ${ }^{61}{ }^{62}$ Innovative cultural and societal approaches will be required to achieve change.

Numerous studies report girls being caught unawares by menarche, and their subsequent feelings of stress, anxiety, depression, dirtiness and anger (eg, see online supplementary table S2.10). While the importance of puberty education is universally recognised, the question of who should educate girls is debated. ${ }^{63}$ India's National Health Mission programme has involved the female community health worker for this purpose with variable results, ${ }^{6465}$ with mothers identified as the most frequent information source in this review. An MHM package thus needs to strengthen mothers' practical MHM knowledge to support girls, while schools teach physiology. Many countries resolve this through age and culture appropriate sex and relationship packages in the school curricula, to familiarise children with puberty changes. ${ }^{66}$

The increasing number of publications on MHM in India is encouraging, showing an interest among researchers in India. Despite the caveats, our data suggest that such studies can provide data relevant for the development of MHM packages which support girls' menstrual needs; they illustrate the need to raise awareness, strengthen life skills education and improve WASH facilities and public health practices.

Contributors AMvE and PAP-H conceived and designed the study. AMvE, MS and $A B$ peformed the literature search and acquired the data. AMvE and PAP-H analysed and interpreted the data. AMvE and PAP-H wrote the first draft of the paper. MS, MBT, SC, $A B$ and KFL critically revised subsequent drafts of the paper. All authors approved the final version.

Funding This review was funded by the Department of Foreign Affairs, Trade and Development (DFAD), Government of Canada through a grant to UNICEF. AMvE had full access to all data and had final responsibility for the decision to submit for publication.

Disclaimer The findings and conclusions in this report are those of the authors and do not necessarily represent the official position of the Centers for Disease Control and Prevention.

\section{Competing interests None declared.}

Provenance and peer review Not commissioned; externally peer reviewed.

Data sharing statement No additional data are available.

Open Access This is an Open Access article distributed in accordance with the Creative Commons Attribution Non Commercial (CC BY-NC 4.0) license, which permits others to distribute, remix, adapt, build upon this work noncommercially, and license their derivative works on different terms, provided the original work is properly cited and the use is non-commercial. See: http:// creativecommons.org/licenses/by-nc/4.0/

\section{REFERENCES}

1. Sommer M, Sahin M. Overcoming the taboo: advancing the global agenda for menstrual hygiene management for schoolgirls. Am J Public Health 2013;103:1556-9.

2. Sommer M. Where the education system and women's bodies collide: the social and health impact of girls' experiences of menstruation and schooling in Tanzania. J Adolesc 2010;33:521-9.
3. Mason L, Nyothach E, Alexander K, et al. 'We keep it secret so no one should know'-a qualitative study to explore young schoolgirls attitudes and experiences with menstruation in rural western Kenya. PLOS ONE 2013;8:e79132.

4. Mahon T, Fernandes M. Menstrual hygiene in South Asia: a neglected issue for WASH (water, sanitation and hygiene) programmes. Gend Dev 2010;18:99-113.

5. McMahon SA, Winch PJ, Caruso BA, et al. 'The girl with her period is the one to hang her head' reflections on menstrual management among schoolgirls in rural Kenya. BMC Int Health Hum Rights 2011;11:7.

6. Adukia A. Sanitation and education. Harvard University, 2014. http:// scholar.harvard.edu/files/adukia/files/adukia_sanitation_and_ education.pdf (accessed 3 Sep 2015).

7. Bodat S, Ghate MM, Majumdar JR. School absenteeism during menstruation among rural adolescent girls in Pune. Natl $J$ Community Med 2013;4:212-16.

8. Sahin M. Tackling the stigma and gender marginalization related to menstruation via WASH in schools programmes. Waterlines 2015;34:3-6.

9. Chothe V, Khubchandani J, Seabert D, et al. Students' perceptions and doubts about menstruation in developing countries: a case study from India. Health Promot Pract 2014;15:319-26.

10. Narayan KA, Srinivasa DK, Pelto PJ, et al. Puberty rituals reproductive knowledge and health of adolescent schoolgirls in south India. Asia Pac Popul J 2001;16:225-38.

11. Thakur $\mathrm{H}$, Aronsson $\mathrm{A}$, Bansode $\mathrm{S}$, et al. Knowledge, practices, and restrictions related to menstruation among young women from low socioeconomic community in Mumbai, India. Front Public Health 2014;2:72.

12. Garg S, Sharma N, Sahay R. Socio-cultural aspects of menstruation in an urban slum in Delhi, India. Reprod Health Matters 2001;9:16-25.

13. Das $\mathrm{P}$, Baker KK, Dutta $\mathrm{A}$, et al. Menstrual hygiene practices, WASH access and the risk of urogenital infection in women from Odisha, India. PLOS ONE 2015;10:e0130777.

14. Sumpter C, Torondel B. A systematic review of the health and social effects of menstrual hygiene management. PLOS ONE 2013;8: e62004.

15. Anand E, Singh J, Unisa S. Menstrual hygiene practices and its association with reproductive tract infections and abnormal vaginal discharge among women in India. Sex Reprod Healthc 2015;6:249-54.

16. Colombia University, UNICEF. MHM in ten: advancing the MHM in WASH in schools agenda. New York, USA: Columbia University and UNICEF, 2014.

17. Sommer M, Vasquez E, Worthington N, et al. WASH in schools empowers girls' education. Proceedings of the Menstrual Hygiene Management in Schools Virtual Conference; 2012. New York, USA: UNICEF and Colombia University, 2013.

18. Alexander K, Oduor C, Nyothach E, et al. Water, sanitation and hygiene conditions in Kenyan rural schools: are schools meeting the needs of menstruating girls? Water 2014;6:1453-66.

19. Prusty RK, Kumar A. Socioeconomic dynamics of gender disparity in childhood immunization in India, 1992-2006. PLoS ONE 2014;9: e104598.

20. Muralidharan A, Patil H, Patnaik S. Unpacking the policy landscape for menstrual hygiene management: implications for school Wash programmes in India. Waterlines 2015;34:79-91.

21. Nyaga VN, Arbyn M, Aerts M. Metaprop: a Stata command to perform meta-analysis of binomial data. Arch Public Health 2014;72:39.

22. Barendregt JJ, Doi SA, Lee YY, et al. Meta-analysis of prevalence. $J$ Epidemiol Community Health 2013;67:974-8.

23. Higgins JP, Thompson SG, Deeks JJ, et al. Measuring inconsistency in meta-analyses. BMJ 2003;327:557-60.

24. Harbord RM, Higgins JP. Meta-regression in Stata. Stata J 2008;8:493-519.

25. Knapp G, Hartung J. Improved tests for a random effects meta-regression with a single covariate. Stat Med 2003;22:2693-710.

26. Arunmozhi R, Antharam P. A cross sectional study to assess the levels of knowledge practices of menstrual hygiene among adolescent girls of Chennai Higher Secondary Schools, Tamil Nadu, 2013. Med ej 2013;3:211.

27. Pokhrel S, Mahantashetti N, Angolkar M, et al. Impact of health education on knowledge, attitude and practice regarding menstrual hygiene among pre-university female students of a college located in urban area of Belgaum. IOSR J Nurs Health Sci 2014;3:38-44.

28. Anuradha R. Menstrual hygiene practices and reproductive morbidity: a community based survey in rural Thiruvananthapuram, 
Kerala. Achutha Menon Centre for Health Science Studies, Sree Chitra Tirunal Institute for Medical Sciences and Technology, 2000.

29. Reddy PJ, Rani DU, Reddy GB, et al. Reproductive health constraints of adolescent school girls. Indian J Soc Work 2005;66:431-40.

30. Nielsen India Private Limited. Baseline research 'Girls today, women tomorrow'-District Jaunpur. New Delhi, India: Nielsen India Private Limited, UNICEF, 2012.

31. Nielsen India Private Limited. Baseline research: 'Girls today, women tomorrow'-District Mirzapur. New Delhi, India: Nielsen India Private Limited, UNICEF, 2012.

32. Nielsen India Private Limited. Baseline research of life skills \& menstrual hygiene behavior among adolescent girls and womenNalanda. New Delhi, India: Nielsen India Private Limited, UNICEF, 2013.

33. Shanbhag D, Shilpa R, D'Souza N, et al. Perceptions regarding menstruation and practices during menstrual cycles among high school going adolescent girls in resource limited settings around Bangalore city, Karnataka, India. Int J Collab Res Intern Med Public Health 2012;4:1353-62.

34. Kumar A, Srivastava K. Cultural and social practices regarding menstruation among adolescent girls. Soc Work Public Health 2011;26:594-604.

35. Ade A, Patil R. Menstrual hygiene and practices of rural adolescent girls of Raichur. Int J Biol Med Res 2013;4:3014-7.

36. Salve SB, Dase RK, Mahajan SM, et al. Assessment of knowledge and practices about menstrual hygiene amongst rural and urban adolescent girls-a comparative study. Int $J$ Recent Trends Sci Technol 2012;3:65-70.

37. Shamima Y, Sarmila M, Nirmalya M, et al. Menstrual hygiene among adolescent school students: an indepth cross-sectional study in an urban community of West Bengal, India. Sudan J Public Health 2013;8:60-4.

38. Khanna A, Goyal RS, Bhawsar R. Menstrual practices and reproductive problems: a study of adolescent girls in Rajasthan. $J$ Health Manag 2005;7:91-107.

39. Nielsen AC. Plan India. Sanitary protection: every woman's health right. New Delhi, India: Nielsen A.C., Plan India, 2010.

40. Oster E, Thornton R. Determinants of technology adoption: peer effects in menstrual cup up-take. J Eur Econ Assoc 2012;10:1263-93.

41. Mason L, Laserson K, Oruko K, et al. Adolescent schoolgirls' experiences of menstrual cups and pads in rural western Kenya: a qualitative study. Waterlines 2015;34:15-30.

42. Emans SJ, Woods ER, Allred EN, et al. Hymenal findings in adolescent women: impact of tampon use and consensual sexual activity. J Pediatr 1994;125:153-60.

43. Nielsen India Private Limited. Baseline research of life skills \& menstrual hygiene behavior among adolescent girls and womenEast Singhbhum. New Delhi, India: Nielsen India Private Limited, UNICEF, 2013

44. Jamwal N. Why are we pretending that there isn't a growing mountain of menstrual waste we need to deal with? 2015. https://in. news.yahoo.com/why-are-we-pretending-that-there-isn-t-a-growingmountain-of-menstrual-waste-we-need-to-deal-with-111659074.html (accessed 28 May 2015).

45. Sahoo P. Ladies, be careful when you throw away the sanitary napkin. 2015. http://www.hindustantimes.com/wellness/ ladies-be-careful-when-you-throw-away-the-sanitary-napkin/ article1-1366417.aspx (accessed 24 Aug 2015)

46. Rihani A. Keeping the promise: five benefits of girls secondary education. Washington DC, USA: AED Global Learning Group, 2006.

47. Mensch BS, Lloyd CB. Gender differences in the schooling experiences of adolescents in low-income countries: the case of Kenya. Stud Fam Plann 1998;29:167-84.
48. National University of Educational Planning and Administration, Department for School Education and Literacy, Ministry of Human Resource Development, et al. Elementary education in India, progress towards UEE. Analytical tables 2012-13. New Delhi, India: National University of Educational Planning and Administration, 2013.

49. ASER Centre. Annual status of education report (Rural) 2014. New Delhi, India: ASER Centre, 2015.

50. Montgomery P, Ryus CR, Dolan CS, et al. Sanitary pad interventions for girls' education in Ghana: a pilot study. PLOS ONE 2012;7:e48274.

51. Oster E, Thornton R. Menstruation, sanitary products and school attendance: evidence from a randomized evaluation. Am Econ $J$ Appl Econ 2011;3:91-100.

52. Freeman MC, Greene LE, Dreibelbis R, et al. Assessing the impact of a school-based water treatment, hygiene and sanitation programme on pupil absence in Nyanza Province, Kenya: a cluster-randomized trial. Trop Med Int Health 2012;17:380-91.

53. Grant MJ, Lloyd CB, Mensch BS. Menstruation and school Absenteeism: evidence from Rural Malawi. Comp Educ Rev 2013:57:260-84.

54. Nallari A. "All we want are toilets inside our homes!" The critical role of sanitation in the lives of urban poor adolescent girls in Bengaluru, India. Environ Urban 2015;27:73-88.

55. Sahoo KC, Hulland KR, Caruso BA, et al. Sanitation-related psychosocial stress: a grounded theory study of women across the life-course in Odisha, India. Soc Sci Med 2015;139:80-9.

56. Gosavi SV, Almale B, Gujarathi A, et al. Awareness and practices about menstrual hygiene and its impact among migrant adolescent girls of Dera: a community based cross-sectional study from Nashik (Maharashtra). Int J Sci Rep 2015;1:123-6.

57. Deeks JJ, Higgins JP, Altman DG. Analysing data and undertaking meta-analyses. In: Higgins JP, Green S, eds. Cochrane handbook for systematic reviews of interventions. The Cochrane Collaboration, 2011:243-96.

58. Tiwari H, Oza UN, Tiwari R. Knowledge, attitudes and beliefs about menarche of adolescent girls in Anand district, Gujarat. East Mediterr Health J 2006;12:428-33.

59. Venkatesh R, Dhoundiyal M. Preceptions and practices during menstruation among adolescent girls in and around Bangalore city. Indian J Matern Child Health 2011;13:1-7.

60. Dugani VS, Angolkar M, Baliga SS, et al. Awareness of menstrual abnormalities amongst pre University College girls (15-18 yrs) in Belgaum, Karnataka State: a cross-sectional study. Indian J Prev Med 2015;3:5-9.

61. Garg S, Anand T. Menstruation related myths in India: strategies for combating it. J Family Med Prim Care 2015;4:184-6.

62. Guterman M, Mehta P, Gibbs M. Menstrual taboos among major religions. Internet J World Health Soc Polit 2007;5:8213.

63. Gupta J, Gupta H. Adolescents and menstruation. J Family Welf 2001;47:1-13.

64. National Rural Health Mission, Ministry of Health and Family Welfare, Government of India. Operational guidelines: promotion of menstrual hygiene among adolescent girls (10-19 years) in rural areas. New Delhi, India: National Rural Health Mission, 2010.

65. Sinha S, Singh A. Adolescent health-tackling menstrual hygiene issue through social marketing of sanitary napkins scheme: an evaluation study from Haryana. J Postgrad Med Educ Res 2013;47:127-30.

66. Department for Education and Employment UK Government. Sex and relationship education guidance. 2000. https://www.gov.uk government/uploads/system/uploads/attachment_data/file/283599/ sex and relationship education guidance.pdf (accessed 24 Aug 2015). 